\title{
Analysis of FSO System and its Challenges - A Review
}

\author{
Priya Sood \\ Research Scholar \\ Sri Sai University \\ Palampur, India
}

\author{
Abhishek Sharma \\ Assistant Professor \\ Sri Sai University \\ Palampur, India
}

\author{
Chandni \\ Assistant Professor \\ Sri Sai University \\ Palampur, India
}

\begin{abstract}
Free Space optics has been emerged as leading communication technology in recent years.. It is an optical communication technology which uses light to propagate the data wirelessly in Free Space. The word 'Free Space' exactly means environment, outer space or vacuum. This technology provides high data rate and can handle large amount of information. It uses the Line Of Sight (LOS) path for transmission. In this paper, discussion about various technologies, different gain and loss occurred during the transmission path. Moreover challenges based on modern and real life applications are then described and also some alternatives have been suggested in order to improve them in future.
\end{abstract}

\section{Keywords}

Optical fiber, free space optics, techniques, challenges, communication

\section{INTRODUCTION}

Communication has become vital part of our day to day routine. Communication means transferring information from one place to another using some medium and this medium plays a significant role. Different services are used to transmit information like voice, video, text, data etc. Now-a-days there is a big demand of these services and also demands of transmission capacity. So for the higher data rate and great bandwidth, light technology has been used[1,2].

Fiber Optics is essential for worldwide Broadband network. With increasing number of users there is a need of wide bandwidth network that can also provide low delay in transmission. Optical fiber provides enormous transmission bandwidth with negligible latency [3]. As they offers numerous advantages such as negligible EMI, high data rate and low carbon footprints optical fibers are used to carry large data transmission for real time communication[4]. FSO employed to handle high data rates in unregulated spectrum. OWC special FSO offers alternate means to communication where signal can only be transferred through free space. The main disadvantage of utilizing FSO is it needs direct Line of Sight for establishing a communication link between transmitter and receiver [5-42.]

In 1970s, prior to the use of FSO was confined to military applications. First full duplex FSO link of $14 \mathrm{Km}$ distance between Yokohama and Tamagawa using the He-Ne Laser of 0.6238 Micrometer was made by Nippon Electric Company[5]. Block diagram of FSO is given below which shows the description of FSO system. Source acts as information that is to be sent through the link. This information is passed on to the modulator where optical carrier; Laser or Led which is the fed into the transmitter unit where this modulated carrier signal along with information signal is passed on to the atmospheric channel. At the receiver end, the information is received and then detected.

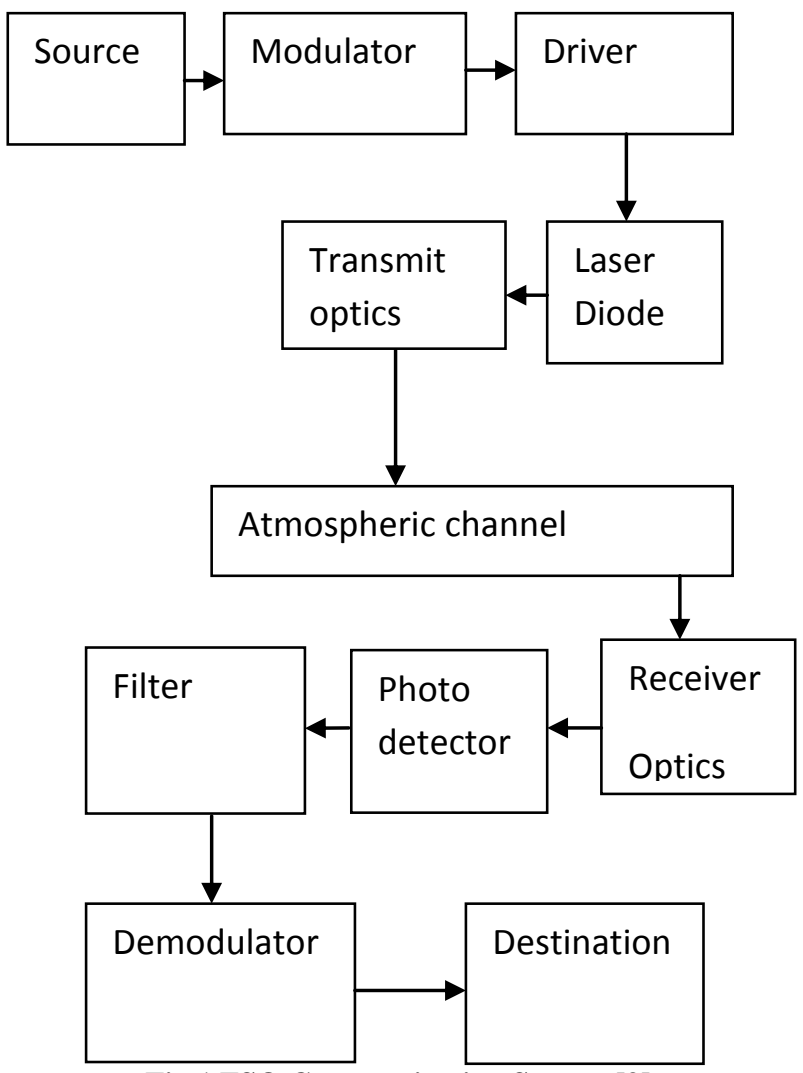

Fig.1 FSO Communication System [9]

\section{CHALLENGES IN FSO}

Absorption and Scattering Loss: The laser beams have to interact with gas molecules and particles present in the atmosphere when it propagate through the earth's atmosphere. The absorption and scattering is the main reason of the loss in atmospheric channel [43-44].

FOG: Due to fog there is an atmospheric attenuation as it results to both absorption and scattering. There will be a high attenuation which is more than $350 \mathrm{db} / \mathrm{km}$ during dense fog conditions [45].

RAIN: The impact of rain is not more like the fog. The drops of rain are larger in size than wavelength used in FSO communication. There is a $2.5 \mathrm{~mm} / \mathrm{hr}$ attenuation loss in light rain and $25 \mathrm{~mm} / \mathrm{hr}$ attenuation loss in heavy rain. [46-47]

SNOW: The size of snow particles are between fog and rain particles. The attenuation due to snow is more than rain but less than fog. The attenuation is ranging between 30$350 \mathrm{db} / \mathrm{km}[48]$.

\section{STUDY RELATED WORK}

In 2007, a study regarding free space communication, which is having high bandwidth. Free space communication is cost effective. In this paper, there is a discussion about the defects which decrease the performance of the link. The turbulence 
induced fading, decrease the performance of the link. To improve the performance, the special diversity can be used over FSO links. The other reason which degrades the performance is rain, fog, snow etc. [49]

In 2010, FSO become more interesting as an alternative to radio frequency communication. In this paper, there is discussed about the gain and loss along the path while the information is sent from source to destination through a medium. There is a need of high speed and tap proof communication system which is useful for free space optical communication. These links were used for various satellites, ground stations etc. These links are mostly used in military and civilians contexts. [51]

In 2011, a study regarding the optical techniques which are used to improve the error rate and distance coverage of free space optical communication system. By using optically amplified and forward (OAF) technique, the received optical field is amplified at each relay. Optically amplified and forward (OAF) increase the BER performance but is degraded by background light. The optically regenerate and forward (ORF) relaying technique is introduced to remove the background noise. By using two equally spaced OAF relays, it increases the communicating distance by $0.9 \mathrm{~km}$ while using two equally spaced ORF relays it increase the range up to 1.9 $\mathrm{km}$. In this paper, there is a comparison between ORF and OAF system by calculating BER, relays, signal to noise ratio, maximum accessible distance. At $\mathrm{BER}=10-5$, these maximum distance $4.5 \mathrm{~km}$ of ORF system is obtained with $\mathrm{M}=2$ relays and $\mathrm{SNR}=29 \mathrm{db}$ which is $1.9 \mathrm{~km}(73 \%)$ more than that of OAF system and also $2.8 \mathrm{~km}(167 \%)$ more than that of direct transmission. [52]

In 2014, a brief survey on free space optical communication is presented. It describes various free space optical channels, transmitter and receiver structures. It provides details on limitation on FSO channels and also suggests some alternatives to improve it. It includes various advances in modulation channel coding, spatial techniques, diversity techniques etc. [53]

In free space optics technology a lot of researches has been done and there is significant improvement in some features which are used to enhance the performance of the system such as data rate, easy deployment, low power requirement etc. however, in spite of these improvements in technology, the performance is degraded by some effects such as absorption, scattering, turbulence etc. In 2016, a paper describes a survey on various challenges faced by FSO for communication between ground to satellite, satellite to ground and inter satellite. It also describes the orbital angular momentum technique in order to provide high capacity for communication links. [54]

A study in 2017, has designed 100gbps free space optical system (FSO) by using optical code division multiple access (OCDMA) technique along with mode division multiplexing (MDM). In this technique, they have used ten channels with data rate of $10 \mathrm{Gbps}$ over a range of $8 \mathrm{~km}$. Random diagonal codes are used. The performance of the system is evaluated under the atmospheric turbulences. Specific results show that, under the clear weather conditions acceptable BER is obtained at a range of $8 \mathrm{~km}$. When there is light fog acceptable BER is obtained over a range of $1500 \mathrm{~m}$. When the light fog is increases then FSO link is obtained over a range f $1250 \mathrm{~m}$ with acceptable BER. On further increasing the fog to heavy level, the results can be obtained only up to $1000 \mathrm{~m}$ with acceptable BER. [55]

\section{CONCLUSION}

Research is a thing that never ends. As the data rate requirements are increasing day by day there is a need to switch from traditional RF systems and Fiber optics to Optical Wireless Communications. Free Space Optics system is unprotected from atmosphere effects like fog, snow, rain etc. Various techniques implemented to resist atmospheric turbulences effects on the quality of received signal. Initially various methods were proposed for FSO communication such as error control codes, modulation, adaptive optics etc. OAF and ORF techniques are used which take advantage of multihop link. By implementing these corrective methods, large capacity of FSO systems can be harnessed and be used in place of existing systems. The advantages of FSO, its bandwidth, security, and its disadvantages such as line of sight requirement, susceptibility to weather etc. Researchers have used different techniques. MDM OCDMA performing the best. It allows ten channels over single FSO link resulting in saving a bandwidth. Thus it is concluded that FSO systems has a very high prospects in near future of communication.

\section{REFERENCES}

[1] Bowman, M., Debray, S. K., and Peterson, L. L. 1993. Reasoning about naming systems.

[2] Ding, W. and Marchionini, G. 1997 A Study on Video Browsing Strategies. Technical Report. University of Maryland at College Park.

[3] Fröhlich, B. and Plate, J. 2000. The cubic mouse: a new device for three-dimensional input. In Proceedings of the SIGCHI Conference on Human Factors in Computing Systems

[4] Tavel, P. 2007 Modeling and Simulation Design. AK Peters Ltd.

[5] Sannella, M. J. 1994 Constraint Satisfaction and Debugging for Interactive User Interfaces. Doctoral Thesis. UMI Order Number: UMI Order No. GAX9509398., University of Washington.

[6] Forman, G. 2003. An extensive empirical study of feature selection metrics for text classification. J. Mach. Learn. Res. 3 (Mar. 2003), 1289-1305.

[7] Brown, L. D., Hua, H., and Gao, C. 2003. A widget framework for augmented interaction in SCAPE.

[8] Y.T. Yu, M.F. Lau, "A comparison of MC/DC, MUMCUT and several other coverage criteria for logical decisions", Journal of Systems and Software, 2005, in press.

[9] Spector, A. Z. 1989. Achieving application requirements. In Distributed Systems, S. Mullender

[10] Sharma, A., Chaudhary, S., Thakur, D., Dhasratan, Vigneswaran "A Cost-Effective High-Speed Radio over Fibre System for Millimeter Wave Applications", Journal of Optical Communications, Published Online: 2017-12-15 | DOI: https://doi.org/10.1515/joc-2017-0166

[11] Sushank Chaudhary, Priyanka Chauhan, Abhishek Sharma, "High Speed 4× 2.5 Gbps-5 GHz AMI-WDMRoF Transmission System for WLANs", Journal of Optical Communications, Published Online: 2017-07-18 | DOI: https://doi.org/10.1515/joc-2017-0082

[12] Sushank Chaudhary, Deepika Thakur, Abhishek Sharma, "10 Gbps-60 GHz RoF Transmission System for 5 G 
Applications", Journal of Optical Communications, Published Online: 2017-07-22 | DOI: https://doi.org/10.1515/joc-2017-0079

[13] Rudrakshi Kapoor, Abhishek Sharma, Sushank Chaudhary, "Empirical Evaluation of 4 QAM and 4 PSK in OFDM-based Inter-Satellite Communication System", Journal of Optical Communications, Published Online: 2017-06-29 | DOI: https://doi.org/10.1515/joc-2017-0059

[14] Abhishek Sharma, Neha Chaudhary, Sushank Chaudhary, " $6 \times 20$ Gbps Hybrid WDM-PI Intersatellite System under the Influence of Transmitting Pointing Errors", Journal of Optical Communications, Published Online: 2016-05-27 | DOI: https://doi.org/10.1515/joc-2015-0099

[15] Abhishek Sharma, Neetu, “ANALYSIS AND MITIGATION OF RECEIVER POINTING ERROR ANGLE ON INTER-SATELLITE COMMUNICATION" International Journal of Innovative Technology and Research, Volume 3, Issue 6, Pages 2540-2544, Nov 2015

[16] Sushank Chaudhary, Abhishek Sharma, Neetu, "6 x 20Gbps Long Reach WDM-PI based High Altitude Platform Inter-Satellite Communication System" International Journal of Computer Applications, Volume 122, Issue 22, Pages 41-45, July 2015

[17] Savita Rana Abhishek Sharma, "Comprehensive study of Radio over Fiber with different Modulation Techniques A Review", International Journal of Computer Applications, Volume 170, Issue 4, Pages 22-25, Aug 2017 DOI: $10.5120 /$ ijca2017914829

[18] Abhishek Sharma, Deepika Thakur, "A Review on WLANs with Radio-Over-Fiber Technology", International Journal of Electronics and Communication Engineering (IJECE), Volume 6, Issue 5, Pages 1-6, Aug 2017.

[19] Kanika Thakur Abhishek Sharma, "Comparison of MDRZ, CSRZ and DRZ schemes using different Communiation Channels", International Journal of Computer Applications, Aug 2017 DOI: 10.5120/ijca2017915106

[20] Kanika Thakur Abhishek Sharma, "Study of Radio over Fiber with Different coding Channel - A Review", International Journal of Computer Applications, Aug 2017, DOI: 10.5120/ijca2017915033

[21] Abhishek Sharma, Priyanka Chauhan, "A Study of Radio over Fiber Technology in WLAN Applications" International Journal for Research in Applied Science \& Engineering Technology (IJRASET) Volume 5 Issue VIII, Page 416-420, August 2017

[22] Abhishek Sharma, Rudrakshi Kapoor, "STUDY OF VARIOUS CHALLENGES IN Is OWC: A Review", International Journal for Research in Applied Science \& Engineering Technology (IJRASET) Volume 5 Issue VIII, Page 802-807, August 2017

[23] Abhishek Sharma, Savita Rana, "Implementation of Radio over Fiber Technology with different filtration techniques" International Journal for Research in Applied Science \& Engineering Technology (IJRASET) Volume 5 Issue VIII, Page 783-789, August 2017.
[24] A. Amphawan and S. Chaudhary, "Free-space optical mode division multiplexing for switching between millimeter-wave picocells," in International Conference on Optical and Photonic Engineering (icOPEN2015), 2015, pp. 95242H-95242H-6.

[25] A. Amphawan, S. Chaudhary, and V. Chan, "2 x 20 Gbps-40 GHz OFDM Ro-FSO transmission with mode division multiplexing," Journal of the European Optical Society-Rapid publications, vol. 9, 2014.

[26] A. Amphawan, S. Chaudhary, R. Din, and M. N. Omar, "5Gbps HG 0, 1 and HG 0, 3 optical mode division multiplexing for RoFSO," in Signal Processing \& Its Applications (CSPA), 2015 IEEE 11th International Colloquium on, 2015, pp. 145-149.

[27] A. Amphawan, S. Chaudhary, T. Elfouly, and K. Abualsaud, "Optical mode division multiplexing for secure Ro-FSO WLANs," Advanced Science Letters, vol. 21, pp. 3046-3049, 2015.

[28] U. Bansal, K. Kaur, and S. Chaudhary, "Role of laser linewidth in high speed DWDM system by incorporating duobinary modulation scheme," International Journal of Computer Applications, vol. 109, 2015.

[29] S. Chaudhary and A. Amphawan, "The role and challenges of free-space optical systems," Journal of Optical Communications, vol. 35, pp. 327-334, 2014.

[30] S. Chaudhary and A. Amphawan, "High-speed millimeter communication through radio-over-freespace-optics network by mode-division multiplexing," Optical Engineering, vol. 56, p. 116112, 2017.

[31] S. Chaudhary, A. Amphawan, and K. Nisar, "Realization of free space optics with OFDM under atmospheric turbulence," Optik-International Journal for Light and Electron Optics, vol. 125, pp. 5196-5198, 2014.

[32] S. Chaudhary, P. Bansal, and M. Lumb, "Effect of beam divergence on WDM-FSO transmission system," International Journal of Computer Applications, vol. 93, 2014.

[33] S. Chaudhary, P. Bansal, and G. Singh, "Implementation of FSO network under the Impact of atmospheric turbulences," International Journal of Computer Applications, vol. 75, 2013.

[34] S. Chaudhary, N. Chaudhary, S. Sharma, and B. Choudhary, "High Speed Inter-Satellite Communication System by Incorporating Hybrid PolarizationWavelength Division Multiplexing Scheme," Journal of Optical Communications.

[35] S. Chaudhary and S. Sharma, "Role of turbulences in WDM-polarization interleaving scheme based intersatellite communication system," International Journal of Computer Applications, vol. 104, 2014.

[36] P. Kaur, R. Kaur, and S. Chaudhary, "Implementation of High Speed Long Reach Hybrid Radio over Multimode Transmission System," International Journal of Computer Applications, vol. 91, 2014.

[37] R. Kaur and S. Chaudhary, "Simulative investigation of laser line-width and channel spacing for realization of DWDM systems under the impact of four wave mixing," Journal of Optical Communications, vol. 35, pp. 157 $165,2014$. 
[38] V. Sharma, "High speed CO-OFDM-FSO transmission system," Optik-International Journal for Light and Electron Optics, vol. 125, pp. 1761-1763, 2014.

[39] V. Sharma and S. Chaudhary, "Implementation of hybrid OFDM-FSO transmission system," International Journal of Computer Applications, vol. 58, 2012.

[40] V. Sharma and S. Kumar, "Empirical evaluation of wired-and wireless-hybrid OFDM-OSSB-RoF transmission system," Optik-International Journal for Light and Electron Optics, vol. 124, pp. 4529-4532, 2013 .

[41] A. Amphawan, S. Chaudhary, and T.-K. Neo, "HermiteGaussian mode division multiplexing for free-space optical interconnects," Advanced Science Letters, vol. 21, pp. 3050-3053, 2015.

[42] K. K. Upadhyay, S. Srivastava, N. Shukla, and S. Chaudhary, "High-Speed 120 Gbps AMI-WDM-PDM Free Space Optical Transmission System," Journal of Optical Communications.

[43] R. K. Long, "Atmospheric attenuation of ruby lasers," Proc. May 1963.

[44] R.M. Langer, "Effects of atmospheric water vapour on near infrared transmission at sea level," in Report on Signals Corps ContractDA-36-039-SC-723351, J.R.M.Bege Co., Arlington, Mass, May 1957.

[45] I. I. Kim and M. Achour, "Free- space links address the last-mile problem," vol. 37, 2001.

[46] A. Z. Suriza, I. M. Rafiqul, A. K. Wajdi, and A. W. Naji, "Proposed parameters of specific rain attenuation prediction for free space optics link operating in tropical region," J. of Atmosp. And SolarTerres. Phys., vol. 94, pp. 93-99, 2013.
[47] A. Vavoulas, H. G. Sandalidis, and D. Varoutas, "Weather effects on FSO network connectivity," J. Opt. Comm. and Net. vol. 4, no.10, pp. 734-740, 2012.

[48] I. I. Kim and E. Korevaar, "Availability of free space optic (FSO) and hybrid FSO/RF systems," Light pointe Tech report, (Weblink:http://www.opticalaccess.com).

[49] S.Mohammad Navidpour, Murat Uysal, and Mohsen Kavehrad, "BER Performance of free space optical transmission with spatial diversity", IEEE TRANSACTIONS WIRELESS COMMUNICATIONS, VOL. 6, NO. 8, AUGUST 2007

[50] Hennes HENNIGER Otakar WILFERT2," An Introduction to Free-space optical communication", RADIOENGINEERING, Vol. 19, No. 2, JUNE 2010

[51] Shabnam kazemlour, Steve Hranilovi, and Shiva Kumar, "All optical multihop free space optical communication system", JOURNAL OF LIGHT WAVE TECHNOLOGY, Vol. 29, No. 18, SEPTEMBER 15, 2011

[52] Hemani Kaushal and Georges Kaddoum,“Optical communication in space: challenges and mitigation techniques", DOI10.1109/COMST.2016.2603518,IEEE Communications Surveys \& Tutorials.

[53] Mohammad Ali Khalighi, Murat Uysal,“Survey on Free Space Optical Communication: A communication Theory perspective", DOI10.1109/COMST.2014.2329501, IEEE communication Surveys and Tutorials.

[54] Himali Sarangal, Amarpal singh , Jyoteesh malhotra, sushank choudhary, "A cost effective 100gbps hybrid MDM-OCDMA-FSO transmission system under atmospheric turbulences", Springer Science Business Media New York 2017 